\title{
Approximate Solution of Schrodinger Equation for Trigonometric Scarf Potential with the Poschl-Teller Non-central potential Using NU Method
}

\author{
C. Cari ${ }^{1,}$ A.Suparmi ${ }^{2}$ \\ 1, 2 (Physics Department, SebelasMaret University, Indonesia)
}

\begin{abstract}
The approximate analytical solution of Schrodinger equation for Scarf potential plus trigonometricPoschl-Teller potential is investigated using Nikiforov-Uvarov method. The bound state energy eigenvalues are given in the close form and the corresponding radial and angular eigenfunctions are formulated in the form of the generalized Jacobi Polynomials. The trigonometric Poschl-Teller potential causes the energy of Scarf potensial decreases as the orbital quantum number increases. The energy spectrum and the radial wave function of Scarf potential are produced by the absent of Poschl-Teller potential.
\end{abstract}

Keywords: Nikiforov-Uvarov method, Schrodinger equation, Trigonometric Scarf I, Poschl-Teller potential.

\section{Introduction}

The exact analytical solution of Schrodinger equations for a class of trigonometric shape invariant potentials are only possible if the angular momentum $1=0$. However, for $\neq 0$, the Schrodinger equation can only be solved approximately for different suitable approximation scheme. One of the suitable approximation scheme is conventionally proposed by Greene and Aldrich.[1-2] The approximate bound state solutions of Schrodinger equation with exponential- line potential such as Eckart potential, Hulthen potential, and Rosen Morse potential have been intensively investigated by some authors.[3-6] It is well known that these solutions play an essential role in the relativistic and non-relativistic quantum mechanics for some physical potentials of interest.

Recently, conciderably efforts have been paid to obtain the exact solution of non-central potentials. There are only a few potentials for which the Schrodinger equation can be solved exactly. In general, one has to resort to numerical techniques or approximation schemes. For many quantum mechanical systems, most approximation methods used are shifted $1 / N$ expansion, WKB method, perturbation method, supersymmetric quantum mechanics and idea of shape invariance, etc. Although some methods produce eigenvalues easily but give complicated eigenfunctions.

In recent years, numerous studies have been made in analyzing the bound state of charged particle moving in a vector potential and a non-central scalar potential, such as an electron moving in a Coulomb field with simultaneously precence of Aharonov-Bohm field[7,8], or/and magnetic monopole[9], Makarov potential [10]or ring-shaped-oscillator potential [11-15], etc. In most of these studies, the eigenvalues and eigenfunctions are obtained using separation variables in spherical coordinate system. Very recently, supersymmetric quantum mechanics with the idea of shape invariance [16-18], factorization method [14-19], and Nikiforov-Uvarov method [20-21] are widely used to derive the energy spectrum and the wave function of a charge particle moving in non-central potential

In this paper we will attempt to solve the Schrodinger equation for trigonometric Scarf potential plus trigonometric Poschl-Teller non-central potential using Nikiforov-Uvarov (NU) method.[20] The trigonometric Scarf potential is also called as generalized Poschl-Teller potential[22]. The NU method, that was developed by Nikiforov-Uvarov [20], was used and applied by reducing the second order differential equations to the hypergeometric type equation by a suitable change of variable.The trigonometric Scarf potential and trigonometric Poschl-Teller play the essential roles in electrodynamics interatomic and intermolecular forces and can be used to describe molecular vibrations. Some of these trigonometric potential are exactly solvable or quasi - exactly solvable and their bound state solutions have been reported.[23-26]

This paper is organized as follows. In section 2, we review the Nikiforov-Uvarov method briefly. In section 3, we find the bound state energy solution and radial wave function from the solution of the radial Schrodinger equation, and the angular wave functions are derived in section 4. A brief conclusion is presented in section 5 . 


\section{Review Of Nikiforov-Uvarov Method}

The one-dimensional Schrodinger equation of any shape invariant potential can be reduced into hypergeometric or confluent hypergeometric type differential equation by suitable variable transformation [3,4,15,17]. The hypergeometric type differential equation, which can be solved using Nikiforov-Uvarovmethod is presented as

$\frac{\partial^{2} \psi(s)}{\partial s^{2}}+\frac{\bar{\tau}(s)}{\sigma(s)} \frac{\partial \psi(s)}{\partial s}+\frac{\bar{\sigma}(s)}{\sigma^{2}} \psi(s)=0$,

where $\sigma(s)$ and $\bar{\sigma}(s)$ are polynomials at most in the second order, and $\bar{\tau}(s)$ is first order polynomial. Equation (1) can be solved using separation of variable method which is expressed as

$\psi=\phi(s) y(s)$,

By inserting equation (2) into equation (1) we get hypergeometric type equation as

$\sigma \frac{\partial^{2} y(s)}{\partial s^{2}}+\tau \frac{\partial y(s)}{\partial s}+\lambda y(s)=0$

and $\phi(s)$ is a logarithmic derivative whose solution is obtained from condition

$\frac{\phi^{\prime}}{\phi}=\frac{\pi}{\sigma}$

where the function $\pi(s)$ and the parameter $\lambda$ are defined as

$\pi=\left(\frac{\sigma^{\prime}-\bar{\tau}}{2}\right) \pm \sqrt{\left(\frac{\sigma^{\prime}-\bar{\tau}}{2}\right)^{2}-\bar{\sigma}+k \sigma}$

$\lambda=k+\pi^{\prime}$

The value of $k$ in equation (5) can be found from the condition that under the square root of equation (5) have to be square of polynomial which is mostly first degree polynomial and therefore the discriminate of the quadratic expression is zero. A new eigenvalue of equation (3) is

$\lambda=\lambda_{n}=-n \tau^{\prime}-\frac{n(n-1)}{2} \sigma^{\prime \prime}, \mathrm{n}=0,1,2$,

where $\tau=\bar{\tau}+2 \pi$

The new energy eigenvalue is obtained using equation (6) and (7).

To generate the energy eigenvalues and the corresponding eigenfunction, the condition that $\tau^{\prime}<0$ is required. The solution of the second part of the wave function, $y_{n}(s)$, is connected to Rodrigues relation ${ }^{[15]}$ which is given as

$y_{n}(s)=\frac{C_{n}}{\rho(s)} \frac{d^{n}}{d s^{n}}\left(\sigma^{n}(s) \rho(s)\right)$

where $C_{n}$ is normalization constant, and the weight function $\rho(s)$ satisfies the condition

$\frac{\partial(\sigma \rho)}{\partial s}=\tau(s) \rho(s)$

The wave function of the system is therefore obtained from equation (4) and (9).

\section{Solution Of Schrodinger Equation For Trigonometric Scarf Potential Combined With Trigonometric Poschl-Teller Potential Using Nu Method}

The non-central potential which is combination of trigonometric Scarf potential and trigonometric Poschl-Teller non-cental potential given as

$V(r, \theta)=\frac{\hbar^{2} \alpha^{2}}{2 M}\left(\frac{b^{2}+a(a-1)}{\sin ^{2} \alpha r}-\frac{2 b\left(a-\frac{1}{2}\right) \cos \alpha r}{\sin ^{2} \alpha r}\right)+\frac{\hbar^{2}}{2 M r^{2}}\left(\frac{\kappa(\kappa-1)}{\sin ^{2} \theta}+\frac{\eta(\eta-1)}{\cos ^{2} \theta}\right)$

where $b^{2}+a(a-1)>0,2 b\left(a-\frac{1}{2}\right)>0, \alpha$ is related to range of the trigonometric Scarf potential with the unit of the inverse of length, $0<\alpha r<\pi, \kappa>1, \eta>1$ and $0 \leq \theta \leq \pi$. Trigonometric Scarf potential is potential model that used to describe the behavior quark-gluon dynamics. ${ }^{[25]}$

The three dimensional time-independent Schrodinger equation for trigonometric Scarf potential $^{[11,14]}$ combined with trigonometric Poschl-Teller non-central potential is

$$
\begin{aligned}
& -\frac{\hbar^{2}}{2 M}\left\{\frac{1}{r^{2}} \frac{\partial}{\partial r}\left(r^{2} \frac{\partial}{\partial r}\right)+\frac{1}{r^{2} \sin \theta} \frac{\partial}{\partial \theta}\left(\sin \theta \frac{\partial}{\partial \theta}\right)+\frac{1}{r^{2} \sin ^{2} \theta} \frac{\partial^{2}}{\partial \varphi^{2}}\right) \psi(r, \theta, \varphi)+\left\{\frac{\hbar^{2} \alpha^{2}}{2 M}\left(\frac{b^{2}+a(a-1)}{\sin ^{2} \alpha r}-\frac{2 b\left(a-\frac{1}{2}\right) \cos \alpha r}{\sin ^{2} \alpha r}\right)+\right. \\
& \hbar 22 M r 2 \kappa \kappa-1 \sin 2 \theta+\eta(\eta-1) \cos 2 \theta \psi r, \theta, \varphi=E \psi r, \theta, \varphi
\end{aligned}
$$

The three dimensional Schrodinger equation expressed in equation (12) is solved using variable separation method by setting $\psi(r, \theta, \varphi)=R(r) P(\theta) \phi(\varphi)$ so we get 
$\frac{1}{R} \frac{\partial}{\partial r}\left(r^{2} \frac{\partial}{\partial r}\right)-r^{2} \alpha^{2}\left(\frac{b^{2}+a(a-1)}{\sin ^{2} \alpha r}-\frac{2 b\left(a-\frac{1}{2}\right) \cos \alpha r}{\sin ^{2} \alpha r}\right)+\frac{2 M r^{2}}{\hbar^{2}} E=-\frac{1}{P \sin \theta} \frac{\partial}{\partial \theta}\left(\sin \theta \frac{\partial}{\partial \theta}\right)-$

$\frac{1}{\phi \sin ^{2} \theta} \frac{\partial^{2}}{\partial \varphi^{2}}+\left(\frac{\kappa(\kappa-1)}{\sin ^{2} \theta}+\frac{\eta(\eta-1)}{\cos ^{2} \theta}\right)=\lambda=l(l+1)$

From equation (13) we get three differential equations with single variable as following:

$\frac{1}{R} \frac{\partial}{\partial r}\left(r^{2} \frac{\partial}{\partial r}\right)-r^{2} \alpha^{2}\left(\frac{b^{2}+a(a-1)}{\sin ^{2} \alpha r}-\frac{2 b\left(a-\frac{1}{2}\right) \cos \alpha r}{\sin ^{2} \alpha r}\right)+\frac{2 M r^{2}}{\hbar^{2}} E=\lambda=l(l+1)$

$-\frac{1}{P \sin \theta} \frac{\partial}{\partial \theta}\left(\sin \theta \frac{\partial}{\partial \theta}\right)-\frac{1}{\phi \sin ^{2} \theta} \frac{\partial^{2}}{\partial \varphi^{2}}+\left(\frac{\kappa(\kappa-1)}{\sin ^{2} \theta}+\frac{\eta(\eta-1)}{\cos ^{2} \theta}\right)=\lambda=l(l+1)$

$\frac{1}{\phi} \frac{\partial^{2}}{\partial \varphi^{2}}=-m^{2}$

The solution of equation (14c) is

$\phi=A_{m} e^{i m \varphi}$

which is the azimuthal part of wave function.

\subsection{The Solution Of Radial Wave Function}

By setting the new wave function $R=\frac{\psi(r)}{r}$ into equation (14a) we get

$\frac{d^{2} \psi}{d r^{2}}-\alpha^{2}\left(\frac{b^{2}+a(a-1)}{\sin ^{2} \alpha r}-\frac{2 b\left(a-\frac{1}{2}\right) \cos \alpha r}{\sin ^{2} \alpha r}\right) \psi+\frac{2 M}{\hbar^{2}} E \psi-\frac{l(l+1)}{r^{2}} \psi=0$

The conventional approximation for centrifugal term ${ }^{[1]}$ obtained for $\alpha r<<<1$ is

$\frac{1}{r^{2}} \cong \alpha^{2}\left(d_{0}+\frac{1}{\sin ^{2} \alpha r}\right)$

where $d_{0}=\frac{1}{12}$. By substituting equation (17) into equation (16) we obtain

$\frac{d^{2} \psi}{d r^{2}}-\alpha^{2}\left(\frac{b^{2}+a(a-1)+l(l+1)}{\sin ^{2} \alpha r}-\frac{2 b\left(a-\frac{1}{2}\right) \cos \alpha r}{\sin ^{2} \alpha r}-l(l+1) d_{0}-\frac{\epsilon^{2}}{\alpha^{2}}\right) \psi=0$

where $\frac{2 M}{\hbar^{2}} E=-\epsilon^{2}$

By making a coordinate transformation, $\cos \alpha r=s$, in equation (18), which is inspired by variable transformation in SUSY WKB ${ }^{[14]}$, then equation (18) becomes

$\left(1-s^{2}\right) \frac{d^{2} \psi(s)}{d s^{2}}-s \frac{d \psi(s)}{d s}-\left\{\frac{b^{2}+a(a-1)+l(l+1)}{1-s^{2}}-\frac{2 b\left(a-\frac{1}{2}\right) s}{1-s^{2}}-l(l+1) d_{0}-\frac{\epsilon^{2}}{\alpha^{2}}\right\} \psi(s)=0$

where for $\alpha r=0$ givess $=1$ and $\alpha r=\pi$ givess $=-1$

By comparing equation (20) with equation (1) we get

$\bar{\tau}=-s, \sigma=1-s^{2}, \quad \bar{\sigma}=-\left\{b^{2}+a(a-1)+l(l+1)-2 b\left(a-\frac{1}{2}\right) s-\frac{\left(1-s^{2}\right)\left(\alpha^{2} l(l+1) d_{0}+\epsilon^{2}\right)}{\alpha^{2}}\right\}$

Inserting equation (21) into equation (5) we have

$\pi=-\frac{s}{2} \pm \sqrt{b^{2}+a(a-1)+l(l+1)-\frac{\alpha^{2} l(l+1) d_{0}+\epsilon^{2}}{\alpha^{2}}+k-2 b\left(a-\frac{1}{2}\right) s+\left(\frac{\alpha^{2} l(l+1) d_{0}+\epsilon^{2}}{\alpha^{2}}-k+\frac{1}{4}\right) s^{2}}$

Since the expression under the square root in equation (22) is quadratic expression with variable $s$ and it has to be square of first degree polynomial, then it can be rewritten as

$\pi=-\frac{s}{2} \pm \sqrt{\frac{\alpha^{2} l(l+1) d_{0}+\epsilon^{2}}{\alpha^{2}}-k+\frac{1}{4}}\left(s-\frac{2 b\left(a-\frac{1}{2}\right)}{2\left(\frac{\alpha^{2} l(l+1) d_{0}+\epsilon^{2}}{\alpha^{2}}-k+\frac{1}{4}\right)}\right)$

and

$\left(2 b\left(a-\frac{1}{2}\right)\right)^{2}-4\left(\frac{\alpha^{2} l(l+1) d_{0}+\epsilon^{2}}{\alpha^{2}}-k+\frac{1}{4}\right)\left(b^{2}+a(a-1)+l(l+1)-\frac{\alpha^{2} l(l+1) d_{0}+\epsilon^{2}}{\alpha^{2}}+k\right)=0$

which is the discriminate of the quadratic expression under the square root. The value of $\mathrm{k}$ can be obtained from the solution of equation (24). By setting

$p^{2}=\frac{\alpha^{2} l(l+1) d_{0}+\epsilon^{2}}{\alpha^{2}}-k+\frac{1}{4}$

equations (23) and (24) become

$\pi=-\frac{s}{2} \pm p\left(s-\frac{2 b\left(a-\frac{1}{2}\right)}{2 p^{2}}\right)$

and $\left(2 b\left(a-\frac{1}{2}\right)\right)^{2}-4 p^{2}\left\{b^{2}+\left(a-\frac{1}{2}\right)^{2}+l(l+1)-p^{2}\right\}=0$

To fulfill the requirement that $\tau^{\prime}<0$ where $\tau=\bar{\tau}+2 \pi$ lead us to make a choice of $\pi$ in equation (26) as 
$\pi=-s\left(p+\frac{1}{2}\right)+\frac{b\left(a-\frac{1}{2}\right)}{p}$

From equation (27) we obtain the values of $\mathrm{p}^{2}$ as

$p_{1}^{2}=\frac{b^{2}+\left(a-\frac{1}{2}\right)^{2}+l(l+1)+\sqrt{\left\{b^{2}+\left(a-\frac{1}{2}\right)^{2}+l(l+1)\right\}^{2}-4\left(b\left(a-\frac{1}{2}\right)\right)^{2}}}{2}$
and $p_{2}^{2}=\frac{b^{2}+\left(a-\frac{1}{2}\right)^{2}+l(l+1)-\sqrt{\left\{b^{2}+\left(a-\frac{1}{2}\right)^{2}+l(l+1)\right\}^{2}-4\left(b\left(a-\frac{1}{2}\right)\right)^{2}}}{2}$

To be physically meaning the value of $\mathrm{p}$ that satisfies the bound state solution is $p_{2}^{2}$ expressed in equation (29b). By inserting the value of $p_{2}^{2}$ in equation (29b) into equation (23) we obtain the $k=\frac{\alpha^{2} l(l+1) d_{0}+\epsilon^{2}}{\alpha^{2}}-p_{2}^{2}+\frac{1}{4}$

By using $\tau=\bar{\tau}+2 \pi$ together with the values of $\pi(\mathrm{s})$ and $\bar{\tau}$ we obtain

$\tau_{2}=-s+2\left\{-s\left(p_{2}+\frac{1}{2}\right)+\frac{b\left(a-\frac{1}{2}\right)}{p_{2}}\right\}=-s\left(2 p_{2}+2\right)+\frac{2 b\left(a-\frac{1}{2}\right)}{p_{2}}$

Now by using the values of $\pi(\mathrm{s}), \bar{\tau}, \mathrm{k}, \sigma(s)$ and $\tau(s)$ we get,

$\lambda=\frac{\alpha^{2} l(l+1) d_{0}+\epsilon^{2}}{\alpha^{2}}+\frac{1}{4}-p_{2}^{2}-p_{2}-\frac{1}{2}$

And $\lambda=\lambda_{n}=-n(-2 p-2)-\frac{n(n-1)}{2}(-2)=2 p n+n+n^{2}$

From equations (32) with (33) we have

Or $\epsilon^{2}=\alpha^{2}\left(p_{2}+n+\frac{1}{2}\right)^{2}-\alpha^{2} l(l+1) d_{0}$

For bound state solution the value of $\mathrm{p}_{2}$ which is chosen from equations (29b) that satisfies equation (34) is

$p=p_{2}=\sqrt{\frac{b^{2}+\left(a-\frac{1}{2}\right)^{2}+l(l+1)-\sqrt{\left\{b^{2}+\left(a-\frac{1}{2}\right)^{2}+l(l+1)\right\}^{2}-4\left(b\left(a-\frac{1}{2}\right)\right)^{2}}}{2}}$

By inserting equations (16) and (35) into equation (34) we get the energy spectra of Scarf potential plus trigonometric Poschl-Teller potential given as

$E_{n}=\frac{\hbar^{2}}{\mathbf{2 M}} \alpha^{2}\left\{\left(\sqrt{\frac{\left(b^{2}+\left(a-\frac{1}{2}\right)^{2}+l(l+1)\right)-\sqrt{\left(b^{2}+\left(a-\frac{1}{2}\right)^{2}+l(l+1)\right)^{2}-4\left(b\left(a-\frac{1}{2}\right)\right)^{2}}}{2}}+n+\frac{1}{2}\right)^{2}-l(l+1) d_{0}\right\}$

For special case, $l=0$, the value of $\mathrm{p}$ is

$p=\sqrt{\left(a-\frac{1}{2}\right)^{2}}=\left(a-\frac{1}{2}\right)$

and so we get the usual energy spectra of trigonometric Scarf potential given as

$E_{n}=\frac{\hbar^{2}}{2 M} \alpha^{2}(a+n)^{2}$

The energy spectrum of the trigonometric Scarf potential for $l=0$ is in agreement with the result derived using Supersymmetric QM. ${ }^{[26,27]}$

The energy eigenfunctions are obtained using equations (4), (9), and (10). By inserting equations (21) and (31) into equation (10) we obtain the weight function $\rho(s)$

and after integrate it we get

$$
(-2 s) \rho+\left(1-s^{2}\right) \rho^{\prime}=\left(s(-2 p-2)+\frac{2 b\left(a-\frac{1}{2}\right)}{p}\right) \rho
$$

$\rho=(1-s)^{\frac{-b\left(a-\frac{1}{2}\right)+p^{2}}{p}}(1+s)^{\frac{b\left(a-\frac{1}{2}\right)+p^{2}}{p}}=(1-\cos \alpha r)^{\frac{-b\left(a-\frac{1}{2}\right)+p^{2}}{p}}(1+\cos \alpha r)^{\frac{b\left(a-\frac{1}{2}\right)+p^{2}}{p}}$

The first part of the energy eigenfunction obtained by inserting equations (21) and (28) into equation (4) is

$$
\begin{aligned}
& \phi(s)=(1-s)^{\left(\frac{-b\left(a-\frac{1}{2}\right)+p^{2}}{2 p}+\frac{1}{4}\right)}(1+s)^{\left(\frac{b\left(a-\frac{1}{2}\right)+p^{2}}{2 p}+\frac{1}{4}\right)}=(1-s)^{\left(\frac{\zeta}{2}+\frac{1}{4}\right)}(1+s)^{\left(\frac{\delta}{2}+\frac{1}{4}\right)} \\
& =(1-\cos \alpha r)^{\left(\frac{\zeta}{2}+\frac{1}{4}\right)}(1+\cos \alpha r)^{\left(\frac{\delta}{2}+\frac{1}{4}\right)} \\
& \text { where } \zeta=\frac{-b\left(a-\frac{1}{2}\right)+p^{2}}{p}=-q+p, \delta=\frac{b\left(a-\frac{1}{2}\right)+p^{2}}{p}=q+p
\end{aligned}
$$


From equations (32) and (41) we get

$$
\begin{gathered}
\zeta=-\sqrt{\frac{\left(b^{2}+\left(a-\frac{1}{2}\right)^{2}+l(l+1)\right)+\sqrt{\left(b^{2}+\left(a-\frac{1}{2}\right)^{2}+l(l+1)\right)^{2}-4\left(b\left(a-\frac{1}{2}\right)\right)^{2}}}{2}}+\sqrt{\frac{\left(b^{2}+\left(a-\frac{1}{2}\right)^{2}+l(l+1)\right)-\sqrt{\left(b^{2}+\left(a-\frac{1}{2}\right)^{2}+l(l+1)\right)^{2}-4\left(b\left(a-\frac{1}{2}\right)\right)^{2}}}{2}(42 \mathrm{a})} \\
\delta=\sqrt{\frac{\left(b^{2}+\left(a-\frac{1}{2}\right)^{2}+l(l+1)\right)+\sqrt{\left(b^{2}+\left(a-\frac{1}{2}\right)^{2}+l(l+1)\right)^{2}-4\left(b\left(a-\frac{1}{2}\right)\right)^{2}}}{2}}+\sqrt{\frac{\left(b^{2}+\left(a-\frac{1}{2}\right)^{2}+l(l+1)\right)-\sqrt{\left(b^{2}+\left(a-\frac{1}{2}\right)^{2}+l(l+1)\right)^{2}-4\left(b\left(a-\frac{1}{2}\right)\right)^{2}}}{2}}
\end{gathered}
$$

(42b)

and

$$
p=\sqrt{\frac{\left(b^{2}+\left(a-\frac{1}{2}\right)^{2}+l(l+1)\right)-\sqrt{\left(b^{2}+\left(a-\frac{1}{2}\right)^{2}+l(l+1)\right)^{2}-4\left(b\left(a-\frac{1}{2}\right)\right)^{2}}}{2}}=\frac{\zeta+\delta}{2}
$$

The second part of the wave function is obtained using Rodrigues relation expressed in equations (9). By substituting equations (21) and (39) into equation (9) we get

$$
\begin{aligned}
& y_{n}(s)=\frac{c_{n}}{(1-s) \frac{-b\left(a-\frac{1}{2}\right)+p^{2}}{p}(1+s) \frac{b\left(a-\frac{1}{2}\right)+p^{2}}{p}} \frac{d^{n}}{d s^{n}}\left((1-s)^{\frac{-b\left(a-\frac{1}{2}\right)+p^{2}}{p}+n}(1+s)^{\frac{b\left(a-\frac{1}{2}\right)+p^{2}}{p}+n}\right) \\
& y_{n}(s)=\frac{C_{n}}{(1-s)^{\zeta}(1+s)^{\delta}} \frac{d^{n}}{d s^{n}}\left((1-s)^{\zeta+n}(1+s)^{\delta+n}\right) \\
& y_{n_{r}}(s)=C_{n_{r}}(-1)^{n_{r}} 2^{n_{r}} n_{r} ! P_{n_{r}}^{(\zeta, \delta)}(s)(43 \mathrm{c}) \\
& \operatorname{and} P_{n}^{(\zeta, \delta)}(\cosh \alpha r)=P_{n_{r}}^{(\zeta, \delta)}(s) \text { is the Jacobi polynomial and } y_{n}(r) \text { is normalized. }
\end{aligned}
$$

From equation (43a) we get the first four of second part of radial wave function or Jacobi polynomials as follows:

$y_{0}(s)=C_{0}$

$y_{1}(s)=C_{1}(-(\zeta+\delta+2) s+(\delta-\zeta))$

$y_{2}(s)=C_{2}\left\{(\zeta+\delta+4)(\zeta+\delta+3) s^{2}-2(\delta-\zeta)(\zeta+\delta+3) s+(\delta-\zeta)^{2}-(\zeta+\delta+4)\right\}$

The radial wave function reduce to

Finally the complete radial wave function obtained by using equation (2), (40), (41) and (42a) is given as

$$
\begin{aligned}
& \psi_{n_{r}}(r)=\psi_{n_{r}}(s)=C_{n_{r}}(-1)^{n_{r}} 2^{n_{r}} n_{r} !(1-s)^{\left(\frac{\zeta}{2}+\frac{1}{4}\right)}(1+s)^{\left(\frac{\delta}{2}+\frac{1}{4}\right)} P_{n_{r}}^{(\zeta, \delta)}(s) \\
& =B_{n_{r}}(1-s)^{\left(\frac{\zeta}{2}+\frac{1}{4}\right)}(1+s)^{\left(\frac{\delta}{2}+\frac{1}{4}\right)} P_{n}^{(\zeta, \delta)}(s)
\end{aligned}
$$

where $B_{n}$ is the new normalisation factor of the radial wave function and it is determined by normalization condition

$\int_{\alpha r=0}^{\alpha r=\pi} \psi_{n_{r}}(r) \psi_{m}(r) d r=\int_{s=-1}^{s=1} \psi_{n_{r}}(s) \psi_{m}(s) \frac{d s}{\alpha \sqrt{1-s^{2}}}=\delta_{n_{r} m}$

Inserting equation (43) into equation (41) we get

$\int_{1}^{-1} B_{n_{r}}(1-s)^{\left(\frac{\zeta}{2}+\frac{1}{4}\right)}(1+s)^{\left(\frac{\delta}{2}+\frac{1}{4}\right)} P_{n}^{(\zeta, \delta)}(s) B_{m}(1-s)^{\left(\frac{\zeta}{2}+\frac{1}{4}\right)}(1+s)^{\left(\frac{\delta}{2}+\frac{1}{4}\right)} P_{m}^{(\zeta, \delta)}(s) \frac{d s}{-\alpha \sqrt{(1-s)(s+1)}}=\delta_{n_{r} m}$

$\frac{\left|B_{n_{r}}\right|^{2}}{\alpha} \int_{-1}^{1}(1-s)^{\zeta}(1+s)^{\delta} P_{n}^{(\zeta, \delta)}(s) P_{n}^{(\zeta, \delta)}(s) d s=1$

Since $\int_{-1}^{1}(1-s)^{\zeta}(1+s)^{\delta} P_{n}^{(\zeta, \delta)}(s) P_{n}^{(\zeta, \delta)}(s) d s=\frac{2^{\zeta+\delta+1} \Gamma\left(n_{r}+\zeta+1\right) \Gamma\left(n_{r}+\delta+1\right)}{\left(2 n_{r}+\zeta+\delta+1\right) \Gamma\left(n_{r}+\zeta+\delta+1\right)}$

then from equation (42) we obtain the normalization constant of the wave function as

$B_{n}=\sqrt{\frac{\alpha\left(2 n_{r}+\zeta+\delta+1\right) \Gamma\left(n_{r}+\zeta+\delta+1\right)}{2^{\zeta+\delta+1} \Gamma\left(n_{r}+\zeta+1\right) \Gamma\left(n_{r}+\delta+1\right)}}$

For $l=0$, equations (42a) and (42b) become

$\zeta=a-\frac{1}{2}-b$ and $\delta=a-\frac{1}{2}+b$

By inserting equation (50) into equation (45) and setting $n_{r}=0$ then equation (45) becomes

$\psi_{0}(r)=B_{0}(1-s)^{\left(\frac{a-b}{2}\right)}(1+s)^{\left(\frac{a+b}{2}\right)}=B_{0}\left(\sin \frac{\alpha r}{2}\right)^{(a-b)}\left(\cos \frac{\alpha r}{2}\right)^{(a+b)}$

Equation (51) is ground state wave function of trigonometric Scarf potential which is in agreement with the result obtained using SUSY quantum mechanics.

The energy eigenvalue expressed in equation (36) is rewritten as 
$E_{n}=\frac{\hbar^{2}}{2 M} \alpha^{2}\left\{\left(\frac{\zeta+\delta}{2}+n+\frac{1}{2}\right)^{2}-l(l+1) d_{0}\right\}$

with $l=\left(\sqrt{\left(\kappa(\kappa-1)+m^{2}\right)}+\eta\right)+2 n_{l}$ which is obtained from the solution of angular Schrodinger equation. The nergy spectrum calculated using equation (52) are listed in Table 2.

The energy eigenvalues in equation (52) and the complete radial wave function expressed in equation (45) both as a function of $\zeta$ and $\delta$ parameters and in turn the $\zeta$ and $\delta$ parameter depend on the value of potential parameters, $a$ and $b$, and also orbital momentum number $l$ therefore both the energy spectrum and the radial wave functions are function s of potential's parameters and orbital quantum number. The radial wave functions reduce to the wave function for Scarf potential plus centrifugal term by the absent of Poschl-Teller potential. The Jacobi polynomials and the radial wave functions with/without the presence of Poschl-Teller potential are listed in Table 1 .

Table 1. The Jacobi Polynomials and Unnormalized Radial Wave Functions for Scarf plus Poschl-Teller Non Central Potential

\begin{tabular}{|c|c|c|c|c|c|c|c|c|c|c|c|}
\hline$n_{l}$ & $m$ & $\kappa$ & $\eta$ & $l$ & $a$ & $b$ & $n_{r}$ & $\frac{\zeta+\delta}{2}$ & $\frac{\delta-\zeta}{2}$ & $y_{n_{r}}(s)$ & $R_{n_{r}}(r)=\phi_{n_{r}} y_{n_{r}}(s)$ \\
\hline 0 & 0 & 0 & 0 & 0 & 2 & 1 & 0 & 1.5 & 1 & 1 & $(1-s)^{(0.5)}(1+s)^{(1.5)}$ \\
\hline 0 & 0 & 0 & 0 & 0 & 2 & 1 & 1 & 1.5 & 1 & $C_{1}(-(5) s+(2))$ & $\begin{array}{c}(1-s)^{(0.5)}(1+s)^{(1.5)} C_{1}(-(5) s \\
+2))\end{array}$ \\
\hline 0 & 0 & 0 & 0 & 0 & 2 & 1 & 2 & 1.5 & 1 & $C_{2}\left(42 s^{2}-24 s-3\right)$ & $\begin{array}{r}(1-s)^{(0.5)}(1+s)^{(1.5)} C_{2}\left(42 s^{2}\right. \\
-24 s-3)\end{array}$ \\
\hline 2 & 0 & 0 & 0 & 4 & 2 & 1 & 1 & 0.32 & 4.8 & $C_{1}(-2.64 \mathrm{~s}+9.6)$ & $\begin{array}{ll}(1-s)^{-2}(1+s)^{2.31} C_{1} & (-2.64 s \\
+9.6) & \\
\end{array}$ \\
\hline$n_{l}$ & $m$ & $\kappa$ & $\eta$ & $l$ & $a$ & $b$ & $n_{r}$ & $\frac{\zeta+\delta}{2}$ & $\frac{\delta-\zeta}{2}$ & $y_{n_{r}}(s)$ & $R_{n_{r}}(r)=\phi_{n_{r}} y_{n_{r}}(s)$ \\
\hline 2 & 0 & 0 & 0 & 4 & 2 & 1 & 2 & 0.32 & 4.8 & $\begin{array}{l}\mathrm{C}_{1}\left(16.9 \mathrm{~s}^{2}-69.9 \mathrm{~s}-\right. \\
87.52)\end{array}$ & $\begin{array}{l}(1-s)^{-2}(1+s)^{2.31} \\
69.9 \mathrm{~s}-87.52)\end{array}$ \\
\hline 2 & 1 & 0 & 0 & 5 & 2 & 1 & 1 & 0.35 & $\begin{array}{l}5.7 \\
6\end{array}$ & $C_{1}(--2.7 \mathrm{~s}+11.52)$ & $\begin{array}{l}(1-s)^{-2.45}(1+s)^{(3.30)} C_{1} \quad(-2.7 \mathrm{~s} \\
+11.52)\end{array}$ \\
\hline 2 & 1 & 2 & 0 & 5.7 & 2 & 1 & 1 & 0.23 & $\begin{array}{l}6.4 \\
3 \\
\end{array}$ & $C_{1}(-2.46 \mathrm{~s}+12.86)$ & $\begin{array}{l}(1-s)^{-2.85}(1+s)^{(3.58)} C_{1}(-2.46 \mathrm{~s} \\
+12.86)\end{array}$ \\
\hline 2 & 1 & 0 & 4 & 9 & 2 & 1 & 1 & 0.16 & $\begin{array}{l}9.6 \\
6\end{array}$ & $C_{1}(-2.32 s+19.32)$ & $\begin{array}{r}(1-s)^{-4.5}(1+s)^{5.16} C_{1}(-2.32 \mathrm{~s} \\
+19.32))\end{array}$ \\
\hline 2 & 1 & 2 & 4 & 9.7 & 2 & 1 & 1 & 7.31 & $\begin{array}{l}7.3 \\
2\end{array}$ & $\mathrm{C}(-16.62 \mathrm{~s}+14.64)$ & $\begin{array}{l}(1 \\
-s)^{(0.24)}(1+s)^{(7.57)} C_{1}(-16.62 s \\
+14.64)\end{array}$ \\
\hline 2 & 0 & 2 & 0 & 5.4 & 2 & 1 & 1 & 0.24 & $\begin{array}{l}6.1 \\
4\end{array}$ & $C_{1}(-2.48 s+12.28)$ & $\begin{array}{l}(1-s)^{-2.7}(1+s)^{3.44} C_{1}(-2.48 s+ \\
12.28)\end{array}$ \\
\hline
\end{tabular}

Table 2. The First and $2^{\text {nd }}$ Excited State Of Scarf

\begin{tabular}{|l|l|l|l|l|l|}
\hline$l$ & $a$ & $b$ & $\frac{\zeta+\delta}{2}$ & $\mathrm{E}_{1}\left(\frac{\hbar^{2}}{2 M} \alpha^{2}\right)$ & $\mathrm{E}_{2}\left(\frac{\hbar^{2}}{2 M} \alpha^{2}\right)$ \\
\hline 0 & 2 & 1 & 1.5 & 4 & 16 \\
\hline 1 & 2 & 1 & 0.69 & 4.63 & 10.01 \\
\hline 2 & 2 & 1 & 0.5 & 3.5 & 8.5 \\
\hline 3 & 2 & 1 & 0.39 & 2.57 & 7.35 \\
\hline 3.4 & 2 & 1 & 0.35 & 2.17 & 6.87 \\
\hline 4 & 2 & 1 & 0.32 & 1.64 & 6.28 \\
\hline 5 & 2 & 1 & 0.35 & 0.92 & 5.62 \\
\hline 5.4 & 2 & 1 & 0.24 & 0.15 & 4.63 \\
\hline 5.7 & 2 & 1 & 0.23 & -0.18 & 4.27 \\
\hline 6 & 2 & 1 & 0.22 & -0.54 & 3.9 \\
\hline 7.4 & 2 & 1 & 0.19 & -2.32 & 2.06 \\
\hline 9 & 2 & 1 & 0.16 & -4.74 & -0.42 \\
\hline 9.7 & 2 & 1 & 0.14 & -5.96 & -2.68 \\
\hline
\end{tabular}


3.2 The Solutionofangularschrodinerequationfor Scarftpotentialcombined Withnon Central PoschlTeller Potential.

The angular Schrodinger equation in equation (14b) is rewritten as

$\frac{1}{\sin \theta} \frac{\partial}{\partial \theta}\left(\sin \theta \frac{\partial P}{\partial \theta}\right)-\frac{m^{2} P}{\sin ^{2} \theta}-\left(\frac{\kappa(\kappa-1)}{\sin ^{2} \theta}+\frac{\eta(\eta-1)}{\cos ^{2} \theta}\right) P+l(l+1) P=0$

wherel $(l+1)$ is separation constant. By making a variable transformation $\cos 2 \theta=x$ in equation (49) the we get

$\left(1-x^{2}\right) \frac{\partial^{2} P}{\partial x^{2}}-\left(\frac{1}{2}+\frac{3}{2} x\right) \frac{d P}{d s}-\left[\left[\frac{2\left(\kappa(\kappa-1)+m^{2}\right)(1+x)}{4\left(1-x^{2}\right)}+\frac{2 \eta(\eta-1)(1-x)}{4\left(1-x^{2}\right)}-\frac{l(l+1)\left(1-x^{2}\right)}{4\left(1-x^{2}\right)}\right]\right] P=0$

The form of equation (50) is similar to the equation (1) thus we have

$\sigma=\left(1-x^{2}\right), \vec{\tau}=-\left(\frac{1}{2}+\frac{3}{2} x\right)$

$\left.\vec{\sigma}=-\frac{\left\{\left\{2\left(\kappa(\kappa-1)+m^{2}\right)+2 \eta(\eta-1)-l(l+1)\right\}\right.}{4}+\frac{\left[2\left(\kappa(\kappa-1)+m^{2}\right)-2 \eta(\eta-1)\right]}{4} x+\frac{l(l+1)}{4} x^{2}\right\}$

Using equations (5) and (51) we obtain

$$
\pi=\left(\frac{1-x}{4}\right) \pm \sqrt{\left\{\frac{2\left(\kappa(\kappa-1)+m^{2}\right)+2 \eta(\eta-1)-l(l+1)}{4}+k+\frac{1}{16}\right\}+\left[\frac{2\left(\kappa(\kappa-1)+m^{2}\right)-2 \eta(\eta-1)}{4}-\frac{1}{8}\right] x+\left(\frac{l(l+1)}{4}-k+\frac{1}{16}\right) x^{2}}
$$

The quadratic expression under the root of equation (52) must be square of first degree polynomial therefore equation (42) can be rewritten as

$\pi=\left(\frac{1-x}{4}\right) \pm \sqrt{\left(\frac{l(l+1)}{4}-k+\frac{1}{16}\right)}\left(x+\frac{\frac{2\left(\kappa(\kappa-1)+m^{2}\right)-2 \eta(\eta-1)}{4}-\frac{1}{8}}{2\left(\frac{l(l+1)}{4}-k+\frac{1}{16}\right)}\right)$

and the discriminate of the quadratic expression under the square root must be zero, that is

$\left[\frac{2\left(\kappa(\kappa-1)+m^{2}\right)-2 \eta(\eta-1)}{4}-\frac{1}{8}\right]^{2}-4\left\{\frac{l(l+1)}{4}-k+\frac{1}{16}\right\}\left\{\frac{2\left(\kappa(\kappa-1)+m^{2}\right)+2 \eta(\eta-1)}{4}+\frac{1}{8}-\frac{l(l+1)}{4}+k-\frac{1}{16}\right\}=0$

To calculate the values of $\mathrm{k}$ in equation (54) we set

$q=\frac{2\left(\kappa(\kappa-1)+m^{2}\right)}{4}$ and $t=\frac{2 \eta(\eta-1)}{4}+\frac{1}{8}=\frac{\left(\eta-\frac{1}{2}\right)^{2}}{2}$

and so from equation (54) we obtain

$k_{1}=\frac{\left(l+\frac{1}{2}\right)^{2}}{4}-\frac{(\sqrt{q}-\sqrt{t})^{2}}{2}$

$k_{2}=\frac{\left(l+\frac{1}{2}\right)^{2}}{4}-\frac{(\sqrt{q}+\sqrt{t})^{2}}{2}$

Therefore from equation (53) and (56) and by imposing the condition that $\tau^{\prime}<0$ we obtain

$\pi_{1}=-x\left(\frac{\sqrt{q}-\sqrt{t}}{\sqrt{2}}+\frac{1}{4}\right)-\frac{\sqrt{q}+\sqrt{t}}{\sqrt{2}}+\frac{1}{4}$ for $k_{1}$

$\pi_{2}=-x\left(\frac{\sqrt{q}+\sqrt{t}}{\sqrt{2}}+\frac{1}{4}\right)-\frac{\sqrt{q}-\sqrt{t}}{\sqrt{2}}+\frac{1}{4}$ for $k_{2}$

By using equations (8), (57a) and (57b) we obtain

$\tau_{1}=-2 x\left(\frac{\sqrt{q}-\sqrt{t}}{\sqrt{2}}+1\right)-2 \frac{\sqrt{q}+\sqrt{t}}{\sqrt{2}}$ for $k_{1}$

$\tau_{2}=-2 x\left(\frac{\sqrt{q}+\sqrt{t}}{\sqrt{2}}+1\right)-2 \frac{\sqrt{q}-\sqrt{t}}{\sqrt{2}}$ for $k_{2}$

By using equations (6), (7), (56), (57), and (58) we obtain the new eigenvalues

$\lambda_{1}=\frac{\left(l+\frac{1}{2}\right)^{2}}{4}-\frac{(\sqrt{q}-\sqrt{t})^{2}}{2}-\left(\frac{\sqrt{q}-\sqrt{t}}{\sqrt{2}}+\frac{1}{4}\right)$ for $k_{1}$

$\lambda_{2}=\frac{\left(l+\frac{1}{2}\right)^{2}}{4}-\frac{(\sqrt{q}+\sqrt{t})^{2}}{2}-\left(\frac{\sqrt{q}+\sqrt{t}}{\sqrt{2}}+\frac{1}{4}\right)$ for $k_{2}$

and

$\lambda_{1}=\lambda_{n}=2 n\left(\frac{\sqrt{q}-\sqrt{t}}{\sqrt{2}}\right)+n(n+1)$ for $\mathrm{k}_{1}$

$\lambda_{2}=\lambda_{n}=2 n\left(\frac{\sqrt{q}+\sqrt{t}}{\sqrt{2}}\right)+n(n+1)$ for $\mathrm{k}_{2}$

The orbital quantum number is obtained from equations (59) and (60) as

$l+\frac{1}{2}= \pm 2\left\{\frac{\sqrt{\kappa(\kappa-1)+m^{2}}-\left(\eta-\frac{1}{2}\right)}{2}+n+\frac{1}{2}\right\}$ for $\mathrm{k}_{1}$

and

$l+\frac{1}{2}= \pm 2\left\{\frac{\sqrt{\kappa(\kappa-1)+m^{2}}+\left(\eta-\frac{1}{2}\right)}{2}+n+\frac{1}{2}\right\}$ for $\mathrm{k}_{2}$

To have physical meaning, the proper choice of $l$ is the values obtained from $\mathrm{k}_{2}$ thus

$l=\left(\sqrt{\left(\kappa(\kappa-1)+m^{2}\right)}+\eta\right)+2 n_{l}$ 
The first part of the wave function obtained by using equations (4) and (53b) is

$\phi=(1-x)^{\frac{\sqrt{q}}{\sqrt{2}}}(1+x)^{\frac{\sqrt{t}}{\sqrt{2}}+\frac{1}{4}}$

The weight function for the second part of the wave function obtained by using equations (10), (51a) and (58b) is

$\rho=(1-x)^{\frac{2 \sqrt{q}}{\sqrt{2}}}(1+x)^{\frac{2 \sqrt{t}}{\sqrt{2}}}$

By using equations (9), (10) and (64) we obtain the second part of the angular wave function given as

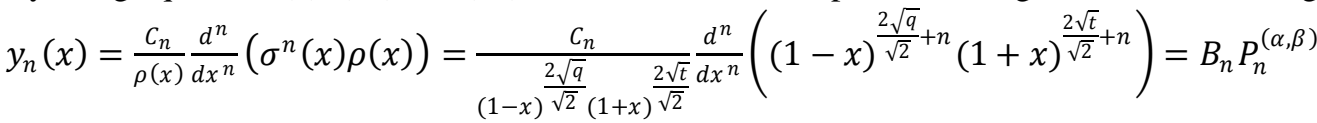

where $P_{n}^{(\alpha, \beta)}$ is the Jacobi polynomial, and

$B_{n}=C_{n}(-1)^{n} 2^{n} n !, \alpha=\sqrt{2 q}, \beta=\sqrt{2 t}$

From equations (2), (63) and (65) we obtain the complete angular wave function as

$P(\theta)=B_{n}(1-x)^{\frac{\alpha}{2}}(1+x)^{\frac{\beta}{2}+\frac{1}{4}} P_{n}^{(\alpha, \beta)}(x)$

The normalization factor $B_{n}$ is obtained from the normalization condition of angular wave function which is expressed in equation (67) as

$\int_{0}^{\pi} P_{m}(\theta) P_{n}(\theta) \sin \theta d \theta=\delta_{m n}$

By inserting equation (67) into equation (68) we have

$-\int_{-1}^{1} B_{m}(1-x)^{\frac{\alpha}{2}}(1+x)^{\frac{\beta}{2}+\frac{1}{4}} P_{m}^{(\alpha, \beta)}(s) B_{n}(1-x)^{\frac{\alpha}{2}}(1+x)^{\frac{\beta}{2}+\frac{1}{4}} P_{n}^{(\alpha, \beta)}(x) \sin \theta \frac{d \theta}{d x} d x=\delta_{m n}$

Since $\frac{d x}{\sin \theta d \theta}=-4 \cos \theta=-4 \sqrt{\frac{1+x}{2}}=-2 \sqrt{2} \sqrt{1+x}$, then equation (69) can be rewritten as

$\frac{B_{m} B_{n}}{2 \sqrt{2}} \int_{-1}^{1}(1-x)^{\alpha}(1+x)^{\beta} P_{m}^{(\alpha, \beta)}(x) P_{n}^{(\alpha, \beta)}(x) d x=\delta_{m n}$

Then the normalization factor obtained from equation (70) is

$B_{n_{l}}=\sqrt{\frac{(2 n+\alpha+\beta+1) n ! \Gamma(n+\alpha+\beta+1)}{2^{\alpha+\beta-\frac{1}{2}} \Gamma(n+\alpha+1) \Gamma(n+\beta+1)}}$

The total wave function of the system is obtained from equations (45), (49), (67), and (71) and is given as

$\psi(r, \theta, \varphi)=B_{n_{r}} D_{n}(1-s)^{\left(\frac{\zeta}{2}+\frac{1}{4}\right)}(1+s)^{\left(\frac{\delta}{2}+\frac{1}{4}\right)} P_{n_{r}}^{(\zeta, \delta)}(s)(1-x)^{\frac{\alpha}{2}}(1+x)^{\frac{\beta}{2}+\frac{1}{4}} P_{n_{l}}^{(\alpha, \beta)}(x)$

Table 3.The First and Second Degree of Jacobi Polynomials with/without The Presence of Non-Central Poschl-

Teller Potential and The Related Un-Normalized Angular Wave Function, $y_{1}(x)$ and $y_{2}(x)$ Obtined from Equation (73b) and (73c)

\begin{tabular}{|c|c|c|c|c|c|c|c|c|}
\hline$n_{l}$ & $m$ & $\kappa$ & $\eta$ & $l$ & $\sqrt{2 q}$ & $\sqrt{2 t}$ & $y_{n_{l}}(x)$ & $P_{l}^{m}(\theta)$ \\
\hline 1 & 0 & 0 & 0 & 2 & 0 & -0.5 & $-1.5 x-0.5$ & $-\left(3 \cos ^{2} \theta-1\right)$ \\
\hline 1 & 1 & 0 & 0 & 3 & 1 & -0.5 & $-2.5 x-1.5$ & $-\sin \theta\left(5 \cos ^{2} \theta-1\right)$ \\
\hline 1 & 0 & 2 & 0 & 3.4 & 1.4 & -0.5 & $-2.9 x-1.9$ & $\sin ^{1.4} \theta(-2.9 \cos 2 \theta-1.9)$ \\
\hline 1 & 0 & 0 & 2 & 4 & 0 & 1.5 & $-3.5 x+1.5$ & $\cos ^{2} \theta(-3.5 \cos 2 \theta+1.5)$ \\
\hline 1 & 0 & 2 & 4 & 7.4 & 1.4 & 3.5 & $-6.9 x+2.1$ & si $n^{1.4} \theta \cos s^{4} \theta(-6.9 \cos 2 \theta+2.1)$ \\
\hline 2 & 0 & 0 & 0 & 4 & 0 & -0.5 & $8.75 x^{2}+2.5 x-3.25$ & $\left\{8.75 \cos ^{2} 2 \theta+2.5 \cos 2 \theta-3.25\right\}$ \\
\hline 2 & 1 & 0 & 0 & 5 & 1 & -0.5 & $\begin{array}{l}15.75 x^{2}+10.5 x \\
-2.25\end{array}$ & $\begin{array}{c}\sin \theta \quad\left\{15.75 \cos ^{2} 2 \theta+10.5 \cos 2 \theta\right. \\
-2.25\}\end{array}$ \\
\hline 2 & 1 & 2 & 0 & 5.7 & 1.7 & -0.5 & $\begin{array}{l}21.84 x^{2}+18.48 x \\
-0.36\end{array}$ & $\begin{array}{c}\text { si } n^{1.7} \theta\left\{21.84 \cos ^{2} 2 \theta+18.48 \cos 2 \theta\right. \\
-0.36\}\end{array}$ \\
\hline 2 & 1 & 0 & 4 & 9 & 1 & 3.5 & $\begin{array}{l}63.75 x^{2}+37.5 x \\
-2.25\end{array}$ & $\begin{array}{c}\sin \theta c o \quad s^{4} \theta\left\{63.75 \cos ^{2} 2 \theta+37.5 \cos 2 \theta\right. \\
-2.25\}\end{array}$ \\
\hline 2 & 1 & 2 & 4 & 9.7 & 1.7 & 3.5 & $\begin{array}{l}75.44 x^{2}+29.52 \mathrm{x} \\
-5.96\end{array}$ & $\begin{aligned} \text { si } \quad n^{1.7} \theta c o \quad s^{4} \theta & \left\{75.44 \cos ^{2} 2 \theta\right. \\
& +29.52 \cos 2 \theta-5.96\}\end{aligned}$ \\
\hline 2 & 0 & 2 & 0 & 5.3 & 1.4 & -0.5 & $\begin{array}{l}19.11 x^{2}+14.82 \mathrm{x}- \\
1.29\end{array}$ & $\begin{array}{c}\text { si } n^{1.4}\left\{19.11 \cos ^{2} 2 \theta+14.82 \cos \theta\right. \\
-1.29\}\end{array}$ \\
\hline 2 & 0 & 0 & 2 & 6 & 0 & 1.5 & $\begin{array}{ll}24.75 & x^{2}-13.5 x- \\
3.25 & \end{array}$ & $\cos s^{2} \theta\left\{24.75 \cos ^{2} 2 \theta-13.5 \cos \theta-3.25\right\}$ \\
\hline
\end{tabular}




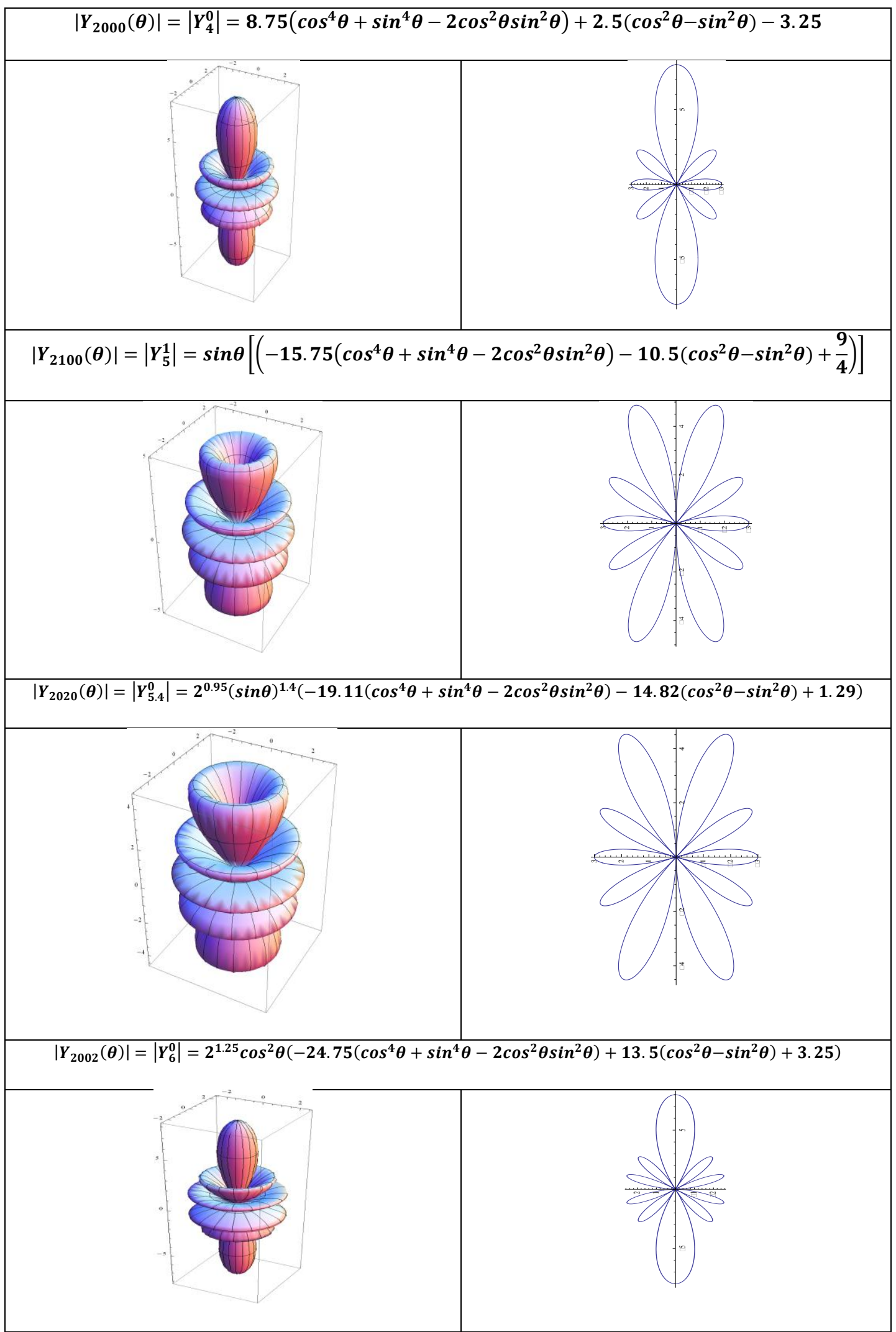

Figure 1. Polar Diagram Of $\left|Y_{l}^{m}\right|$ With/Without The Presence of Poschl-Teller Potential and The Corresponding The Three Dimensional Polar Representations of Absolute Values of Angular Function. The Effect Of cosec ${ }^{2} \theta$ Is Similar With The Effect of M, Magnetic Quantum Number Which Change The Direction of Angular Momentum In Z Direction, As Shown In Figures (B) And (C), While The Effect of $\sec ^{2} \theta$ 
Construction of Jacobi polynomials

The first three un-normalized Jacobi polynomials obtained from equation (65) are

$y_{0}(x)=C_{0}(73 a)$

$y_{1}(x)=C_{1}[-\sqrt{2 q}-\sqrt{2 t}-2] x+(\sqrt{2 t}-\sqrt{2 q})$

$y_{2}(x)=[\sqrt{2 q}+\sqrt{2 t}+4][\sqrt{2 q}+\sqrt{2 t}+3] x^{2}-2(\sqrt{2 t}-\sqrt{2 q})[\sqrt{2 q}+\sqrt{2 t}+3] x+(\sqrt{2 t}-\sqrt{2 q})^{2}-$

$[\sqrt{2 q}+\sqrt{2 t}+4](73 \mathrm{c})$

The first and second order of Jacoby polynomials with and without the presence of Poschl-Teller potential determined analytically using equations (73b) and (73c) and its corresponding polar wave function are presented in Table 2. By the absent of Poschl-teller potential the angular wave functions reduce to associated Legendre function but only for which the different values of $l$ and $m$ are even number as shown in the $1^{\text {st }}, 2^{\text {nd }}, 6^{\text {th }}$ and $7^{\text {th }}$ rows. The effect of the presence of Poschl-Teller potential is described using the polar diagram of the angular wave function and three dimensional representation of the absolute value of the angular wave function as shown in Figure 1. The only significant effect of the presence of Poschl-Teller potential to the angular wave function is coming from the $s e c^{2} \theta$ term as shown in figure 1 (d). The $s e c^{2} \theta$ term causes the increase of the orbital quantum number thus change the degeneracy number.[28,29]

\section{Result And Discussion}

The presence of Poschl-Teller non-central potential simultaneously with Scarf potential causes increases the orbital quantum number and decreases the energy levels of Scarf potential. By setting $\eta=\kappa=$ $l=0$ the energy spectrum and the radial wave function of the non-central potential reduces to the energy spectrum and wave function of Scarf potential. For $\eta=\kappa=0$ the angular wave function of Scarf potential with Poschl-Teller reduces to associated Legendre polynomials with $l$ and $m$ differed by even numbers. Both the radial or angular wave functions are expressed in Jacobi polynomials and the angular wave function is normalized. It may be concluded that there is an effect of the presence of Poschl-Teller potential to the system governed by Scarf potential, therefore the Poschl-Teller non-central potential can be considered as the perturbation part.

\section{Conclusion}

It has been shown that the Schrodinger equation of Scarf potential with Poschl-Teller non-central potential is solved approximately using Nikiforov-Uvarov method with suitable coordinate transformation. The energy spectrum of the system is obtained in the closed form and the radial and angular wave function is expressed in Jacobi polynomials. The presence of Poschl-Teller non-central potential causes the decrease in energy spectrum of Scarf potential and increases the orbital quantum number.

\section{Acknowledgements}

This work is partially supported by Hibah Pascasarjana Sebelas Maret University grant No. 2910/UN27.10/PG/2012.

\section{References}

[1] R.I. Greene and C. Aldrich, Variational wave function for a screened Coulomb potential, Phys. Rev. A 14(6), (1976) 2363

[2] S.M. Ikhdair and R. server, Approximate eigenvalue and eigen function solutions for the generalized Hulthen potential with any angular momentum, J. Math. Chem., 42(3) (2007) 461-471

[3] A.N. Ikot and L.E.Akpabio, Approximate solution of the Schrodinger solution with Rosen-Morse potential including the centrifugal term, App. Phys. Res, 2(2) (2010) 202-208

[4] H. Goudarzi and V. Vahidi, Supersymmetric approach for Eckart potential using NU method,Adv. Studies Theor. Phys. 5(10), (2011) 469-476

[5] A.R. Amani, M.A. Moghrimoazzen, H. Ghorbanpour, S. Barzegaran, The ladder operators of Rosen Morse potential with centrifugal term by factorization method, Afr. J. Math. Phys. 10 (2011) 31-37

[6] S.M. Ikhdair, Approximate 1-state of the Manning-Rosen potential by using NU method, ISRN Math. Phys. vol 2012,article ID201525 (2012)

[7] V.M. Villalba, Elsevier Science, Bound State of The Hydrogen Atom in The Presence of A Magnetic Monopole Field and An Ahoronov-Bohm Potential, Phys. Lett. A 193 (1994) 218-222

[8] Y. Aharonov and D. Bohm, Significance of electromagnetic potentials in quantum theory, Phys. Rev. 115 (1959) 485

[9] L. V. Hoang, L.X. Hai, L.I. Komarov and T. S. Romanova, Relativistic Analogy of the Aharonov-Bohm Effect in the Presence of Coulomb Field and Magnetic Charge, J. Phys. a 25 (1992) 6461

[10] M. Movahedi and M. Hamzavi, Relativistic scattering solution of the Makarov potential, Int. J. phys. Sci. , 6(4) (2011) 891-896

[11] S. Ferrer, A unified treatment for some ring-shaped potentials as a generalized 4-D isotropic oscillator, Mono. Acad. Ci. Zar, Vol. 30 (2006) 11-21

[12] C.Y Chen and S. H. Dong,Exactly complete solutions of the Coulomb potential plus a new ring-shaped potential, Phys. Lett. A 335 (2005) 374

[13] H.X. Quan, L. Guang, W.Z. Min, N.L. Bin, and M. Yan, Solving Dirac Equation with New Ring-Shaped Non-Spherical Harmonic Oscillator Potential, Com. Theor. Phys. (China) 53 (2010) 242 
[14] J. Sadeghi and B. Pourhassan, Exact solution of the non-central modified Kratzer potential plus a ring-shaped like potential by the factorization method, EJTP, 5, 193 (2008).

[15] D. Agboola, Schrödinger Equation with Hulth'en Potential Plus Ring-Shaped Potential, Commun. Theor. Phys. 55 (2011) $972-976$

[16] B. Gonul and I. Zorba, Supersymmetric Solutions of Non-Central Potentials, Phys. Lett. A 269 ( 2000) 83

[17] J.C.Sheng, Z.Ying, Z.X. Lin, S.L.Tian, Identity for the Exponential-Type Molecule Potentials and the Supersymmetry Shape Invariance, Com. Theor. Phys. 36 (2001) 641-646

[18] A.Khare and R.K. Badhuri, Spersymmetry shape invariance and exactly solvable non-central potential, arXiv:hep-th/9310104v1 (1993)1-16

[19] J. Sadeghi,Factorization Method and Solution of the Non-Central ModifedKratzer Potential, Act. Phys. Polonica, A 112 (1) (2007) 23

[20] A. F. Nikiforov and U. B. Uvarov, Special Function in Mathematical Physics, Birkhausa, Basel (1988)

[21] A. P. Raposo, H. J. Weber, D. E. Alvarez-Castillo, and M. Kirchbach,Romanovski Polynomials in Selected Physics Problems,arXiv:0706.3897v1 [quant-ph] 26 Jun 2007

[22] J. Derezinski and M. Wrochna, Exactly solvable Schrodinger operator, arXiv: 10090541v2 (math-ph) (2010) 1- 20Y. Xu, S. He and C.S. Jia, Approximate analytical solutions of the Klein-Gordon equation with the P"oschl-Teller potential including the centrifugal term, Phys. Scr. 81 (2010) 045001

[23] A.H. El Kineni and M. Daoud, Coherent States a la Klaude-Perelomov for the Poschl-Teller potentials, Phys. Lett. A 283 (2001) 291-299

[24] MetinAktas, Exact solutions to a new generalized non-central potential in three dimensions, arXiv: quant-ph /0701063v1 (2007)

[25] H. Salehi, Determine the eigen function of Schrodinger equation with non-central potential by using NU method, App. Math. 2 (2011) 999-1004

[26] D.E. Alvarez -Castillo, C. B. Compean, M. Kirchbach, Rotational Symmetry and Degeneracy: A Cotangent-Pertubed Rigid Rotator of Unpertubed Level Multiplicity, arXiv: 1105.1354v1[quant-ph] (2011)1-10

[27] D.E Alvarez -Castillo and M. Kirbach, Exact Spectrum and Wave Functions of The Hyperbolic Scarf Potential in Terms of Finite Romanovski Polynomials,Rev. Mex. Fis. E53.2 (2007) 143-154

[28] Flugge, Practical Quantum Mechanics, I (1977) 